Melly Ludovic (Orcid ID: 0000-0002-0951-269X)

\title{
Fibrin hydrogels promote scar formation and prevent therapeutic angiogenesis in the heart
}

\author{
Fibrin hydrogels promote myocardial scar formation
}

L. Melly ${ }^{1,2}$, A. Grosso ${ }^{2}$, C. Stanciu Pop ${ }^{3}$, C. Yu-Hsuan ${ }^{4}$, MC. Nollevaux ${ }^{3}$, C. Schachtrup ${ }^{4}$, A. Marsano ${ }^{5}$, N. Di Maggio ${ }^{2}$, B. Rondelet ${ }^{1 *}$, A. Banfi $^{2 *}$

1 Cardiac, Vascular and Thoracic Surgery Department, CHU UCL Namur, Yvoir, Belgium.

2 Cell and Gene Therapy, Department of Biomedicine, Basel University Hospital and University of Basel, Basel, Switzerland.

3 Pathology Department, CHU UCL Namur, Yvoir, Belgium.

4 Institute of Anatomy and Cell Biology, Department of Molecular Embryology, University of Freiburg, Germany.

5 Cardiac Tissue Engineering, Department of Biomedicine, Basel University Hospital and University of Basel, Basel, Switzerland.

* Equally contributing authors

\section{Correspondence to:}

Dr. Ludovic MELLY (e-mail: ludovic.melly@uclouvain.be)

or Dr. Andrea BANFI (e-mail: andrea.banfi@usb.ch).

Word count: 5802

Key Words: Fibrin; Hydrogel; VEGF; PDGF; Intramyocardial injection; Rat.

This article has been accepted for publication and undergone full peer review but has not been through the copyediting, typesetting, pagination and proofreading process which may lead to differences between this version and the Version of Record. Please cite this article as doi: $10.1002 /$ term.3118 


\section{Abstract}

Therapeutic angiogenesis is the delivery of factors to promote vascular growth and holds promise for the treatment of ischemic heart conditions. Recombinant proteindelivery to the myocardium by factor-decorated fibrin matricesis anattractive approach, thanks to the abilityto precisely control both dose and duration of the treatment, the use of a clinically approved material like fibrin and the avoidance of genetic modification. Here we investigated the feasibility of inducing therapeutic angiogenesis in the rat myocardium by a state-of-the-art fibrin-based delivery platform that we previously optimized.Engineered versions of murine VEGF 164 and PDGF-BB were fused with an octapeptide substrate of the transglutaminase coagulation factor fXIIIa(TG) to allow their covalent cross-linking into fibrin hydrogels and release by enzymatic cleavage. Hydrogelscontaining either $100 \mu \mathrm{g} / \mathrm{ml}$ TGVEGF alone or in combination with $10 \mu \mathrm{g} / \mathrm{ml}$ TG-PDGF-BB or no factor were injected into rat myocardium. Surprisingly, vascular density was severely reduced in all conditions, both in and around the injection site, where large fibrotic scars were formed. Scar formation was not due to the presence of growth factors, adaptive immunity to human proteins, damage from injection, nor to mechanical trauma from the hydrogel stiffness or volume. Rather scar was induced directly by fibrin and persisted despite hydrogel degradation within 1 week. These results caution against the suitability of fibrin-basedplatforms for myocardial growth factor delivery, despite their efficacy in other tissues, like skeletal muscle. The underlying molecular mechanisms must be further investigated in order to identify rational targets to prevent this serious side-effect.

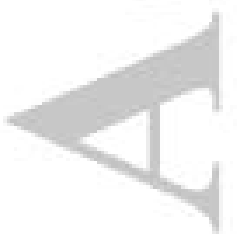




\section{Introduction}

Ischemic heart disease still remains one of most frequent causes of death worldwide.Therapeutic angiogenesis promotes expansion of microvascular networks, which can lead to increased flow also in upstream collateral arteries (arteriogenesis) through retrograde signals(Annex, 2013), thereby providing effective bypass of the obstructed feeding vessels. Thus stimulation of microvascular growth has the potential to treatseveral ischemic conditions, either alone or in combination with revascularization strategies, such as percutaneous angioplasty or coronary artery bypass grafting.

Vascular Endothelial Growth Factor-A (VEGF) is the master regulator of angiogenesis(Carmeliet \& Jain, 2011) and its combination with Platelet-Derived Growth Factor-BB (PDGF-BB) has been shown to provide optimal stimulation of both angiogenesis and arteriogenesis(Gianni-Barrera et al., 2016). However, VEGF can also induce aberrant and dysfunctional vascular growth if its distribution and duration of expression are not carefully controlledin vivo(Gianni-Barrera et al., 2020). In fact, since VEGF binds tightly to the extracellular matrix, different levels of expression do not average in tissue, but rather remain localized in the microenvironment around each producing cell(Ozawa et al., 2004). Cell-based gene therapy approaches, whereby progenitor populations are genetically engineered and FACS-purified to homogenously produce desired levels of the genes of interest(Misteli et al., 2010), have been show to enable precise control of VEGF dose distribution in vivo and ensure only physiological and functional angiogenesis in the myocardium (L. F. Melly et al., 2012)(L. Melly et al., 2018). However, the clinical application of such approaches is challenging due to both the difficulty to achieve sufficient expansion of the transduced populationsand the safety issues concerning stable genetic transduction of progenitors (Baum et al., 2003). On the other hand, gene therapy vectors (e.g. 
adenoviruses) have been the most investigated tool for clinical trials of therapeutic angiogenesis because they afford two very desirable features: a) efficient gene transfer with robust expression, and b) transient duration that is limited by theimmune response. However, these same features compromise safety(by uncontrolled distribution of high levels) and efficacy (by regressionof unstable vessels).

Controlled delivery of recombinant protein factors from bio-compatible matriceshas recently emerged as a clinically attractive approach to control both the dose and duration of treatment, while ensuring a homogeneous distribution in vivo, therefore improving both safety and efficacy (Martino et al., 2015).We previouslyoptimized a fibrin-based platform for the controlled delivery of recombinant VEGF, engineered to contain an octapeptide sequence that is a substrate for the transglutaminase factor XIIla (TG-VEGF)(Sacchi et al., 2014). This modification allows its cross-linking into a fibrin hydrogelduring fibrinogen coagulation and ensures its controlled release only by cell-demanded enzymatic cleavage. Fibrin is a natural product of blood coagulation and provides unique features for physiological presentation of angiogenic signals. Fibrin is also injectable as a liquid, becomes a hydrogelin situ without cytotoxicity, and last also provides the universalmatrix for tissue regeneration after damage. We previously showed that controlled VEGF delivery by this optimized factor-decorated fibrin matrix was therapeutically effective in rodent models ofhind limb ischemia and wound healing (Sacchi et al., 2014).

Because of its desirable clinical features, fibrin-based hydrogels have been used in several studies of cell therapy to repair the injured heart, either by intramyocardial injection (Christman, Fok, Sievers, Fang, \& Lee, 2004; Christman, Vardanian, et al., 2004; Ryu et al., 2005) or as an epicardial patch (Godier-Furnemont et al., 2011; Xiong et al., 2011; Ye et al., 2014).However, controlled and sustained delivery of recombinant therapeutic factors 
covalently incorporated in a fibrin hydrogel to the heart has not been attempted. Therefore, here we investigated the feasibility and safetyof factor-decorated fibrin matrices to deliver an optimal combination of the angiogenic and arteriogenic factors VEGF and PDGF-BB to the myocardium of immune-competent normal rats.

\section{Methods}

\section{Gel preparation}

Fibrin matrices were prepared by mixing human fibrinogen in different concentrations (2.5 to $25 \mathrm{mg} / \mathrm{mL}$; Enzymes Research Laboratories), human factor XIIla (2 U/mL; CSL Behring), human thrombin ( $2 \mathrm{U} / \mathrm{mL}$; Sigma-Aldrich) and if needed TG-aprotinin $(56 \mu \mathrm{g} / \mathrm{mL})$, combined with murineTG-VEGF 164 alone $(100 \mu \mathrm{g} / \mathrm{mL})$ or together with murineTG-PDGF-BB (10 $\mathrm{\mu g} / \mathrm{mL})$ and $2.5 \mathrm{nM} \quad \mathrm{Ca}^{+2}$ in $\quad$ 4-(2-hydromethyl)-1-piperazineethanesulfonic acid (Hepes).Fibrinogen and both enzymes were purified from plasma of healthy donors. Fibrin gels were created with the highest feasible dose of TG-VEGF to ensure maximum arteriogenic potential, in a ratio of $10: 1$ with transglutaminase-PDGF-BB (V: $100 \mu \mathrm{g} / \mathrm{mL}$ and P: $10 \mu \mathrm{g} / \mathrm{mL}$ ) based on previous results. Matrices containing TG-aprotininand TG-VEGF164 were obtained by adding the engineered proteins to the cross-linking enzymes solution before mixing with fibrinogen. Before injection the two components were mixed to allow in situ polymerization. For the degradation trial the unlabeled fibrinogen $(25 \mathrm{mg} / \mathrm{mL})$ was mixed with a 647-flurochrome marked fibrinogen $(0.5 \mathrm{mg} / \mathrm{mL}$; Invitrogen).

\section{In vivo gel injection}

Animals were treated in compliance with Swiss Federal guidelines for animal welfare and all procedures were approved by the Veterinary Office of the Canton Basel (Basel, 
Switzerland) and conform to the Directive 2010/63/EU of the European Parliament. Sprague-Dawley (RjHan:SD) or nude (Crl: NIH-Foxn1 ${ }^{\text {rnu }}$, Charles River) rats were anesthetized with $2.5 \%$ isoflurane and $10 \mathrm{mg} / \mathrm{kg}$ buprenorphine with tracheal ventilation at $80 \mathrm{cycles} / \mathrm{min}$ (Small Animal Ventilator 683, Harvard Apparatus). The heart was exposed through a left thoracotomy. The gel was injected into four sites of the anterior wall for a total volume of $100 \mu \mathrm{L}$. At sacrifice, rats were anesthetized under the same anesthesia conditions as already described. Hearts were perfusedin a retrograde manner through the descending aorta with paraforamaldehyde $0.5 \%$ for three minutes. Hearts werethen collected, embedded in OCT compound (Sakura Finetek), and frozen in isopentane cooled in liquid nitrogen.

\section{Tissue staining}

Histological analyses were performed on heart cryosections. Masson Trichrome staining was performed according to standard protocols. Immunofluorescence was performed using the following primary antibodies and dilutions: mouse anti-rat CD31 (Clone TLD-3A12; AbDSerotec; $1: 100)$, mouse anti- $\alpha$-smooth muscle actin (Clone 1A4; MPBiomedicals; 1:400), rabbit anti-PDGF Receptor $\alpha$ (Clone D1E1E; Cell Signaling; 1:500), goat anti-mouseCD206 (R\&D Systems; 1:1000). Fluorescently labeled secondary antibodies (Invitrogen) were used at 1:200. Immunohistochemestry was performed using the rabbit-anti-rat CD31 (Clone EPR17259; Abcam; 1:1000).

\section{Vessel and scar measurements}

The amount of blood vessels was quantified on images taken from CD31-stained sections. Representative images on immunohistochemistry staining were acquired with a $20 \mathrm{x}$ objective on aLeicaDM 2002 LED microscope on cross-sections of the anterior wall, as well 
as the border zone and the contralateral myocardium. Six different injection sites per group (with each one averaged two quantifications per injection) were quantified by using the absolute number of CD31-positive structures on a given circular area of $100 \mu \mathrm{m}$ in diameterand then normalized per $\mathrm{mm}^{2}$. Measures were made within the injection site, on the border zoneand in the remote contralateral myocardium of the same section. For the maximal scar area, six representative images with per injection per group, acquired with a $2.5 x$ objective, were chosen among 8 sections per animal taken at $300-\mu \mathrm{m}$ intervals. Blind analysis of the maximal scar area was calculated using the Fiji software (BSD license).

\section{Statistics}

Data are presented as mean \pm standard deviation and were analyzed with the statistical software Prism 8 (GraphPad) for significance of differences. The normal distribution of all data sets was tested and, depending on the results, multiple comparisons were performed with the parametric one-way analysis of variance (ANOVA) followed by the Tukey test, or with the nonparametric unpaired t test. $p<0.05$ was considered statistically significant.

\section{Results}

\section{Intramyocardial injection of fibrin hydrogels is feasible}

The liquidfibrinogen mixtures completely polymerized within 60 to 90 seconds after mixing with cross-linking enzymes. This time allowed successful injection into the front wall of the left ventricle in all 50 animals, which had an average weight of $349 \pm 128 \mathrm{~g}$. No neurological deficits were observed, indicating the absence of intra-ventricular injections and potential embolizationto the brain. Peri-procedural mortality remained null and all animals could complete their planned time-point without side-effects. 
Fibrin hydrogels severely reduce vascular density regardless of angiogenic factor decoration

In order to assess the angiogenic potential of factor-decorated fibrin matrices, hydrogels were decorated either with $100 \mu \mathrm{g} / \mathrm{ml}$ of TG-VEGF alone (V) or in combination with 10 $\mu \mathrm{g} / \mathrm{ml}$ of TG-PDGF-BB (VP) and injected in the left ventricle myocardium as 4 individual volumes of $25 \mu$ Leach.Empty fibrin hydrogels without any growth factor served as negative control (Ctr). These doses of the VP combination were previously determined to provide the maximum angiogenic and arteriogenic effect in skeletal muscle (Sacchi et al. unpublished results).Surprisingly, only sparse vascular structures were found within the injection sites in all groups, regardless of angiogenic factor presence $(\mathrm{Ctr}=772 \pm 130, \mathrm{~V}=746 \pm 316, \mathrm{VP}=$ $830 \pm 259 \mathrm{CD} 31+$ structures $/ \mathrm{mm}^{2}, p=n . s$.). Not only no angiogenic effect was observed in the factor-decorated hydrogels compared to fibrin alone, but the vascular density in all injection sites was severely reduced compared to the immediately adjacent border zones(Ctr = $2515 \pm 371, V=2410 \pm 359, V P=2897 \pm 342 \mathrm{CD} 31+$ structures $/ \mathrm{mm}^{2}, \mathrm{p}<0.0001$ vs the respective injection sites)and even more compared to normal myocardium in the remote posterior wall of the ventricle $\left(\mathrm{Ctr}=3677 \pm 510, \mathrm{~V}=3714 \pm 287, \mathrm{VP}=4074 \pm 128 \mathrm{CD} 31+\right.$ structures $/ \mathrm{mm}^{2}$, $p<0.0001$ vs both the respective injection sites and border zones) (Figure 1). On the other hand, the reduction of vascular density in the injection sites was accompanied by the infiltration of anamorphous cellularizedtissue with abundant extracellular matrix in place of the normal cardiomyocyte fibers (Fig. 1A).

Fibrin hydrogels induce a fibrotic scar similar to myocardial infarction

Masson Trichrome staining revealed that the newly formed tissue within the injection sites displayed the typical features of a fibrotic scar and was similarly composed of abundant collagen fibers in all three groups (Fig. 2A). To determine whether the scar could 
be due to tissue damage induced by the injection, a similar volume of phosphate-buffered saline (PBS) was injected using the same 30G syringe (Fig. 2B).Quantification of the collagen area after Masson Trichrome stainingrevealed that all three fibrin-containing treatments induced a significantly larger scar than PBS after 1 week(Fig. $2 \mathrm{C}, \mathrm{Ctr}=1.31 \pm 0.36$; $\mathrm{V}=$ 1.16 $\pm 0.20 ; \mathrm{VP}=0.76 \pm 0.24 \mathrm{~mm}^{2}$ vs $\mathrm{PBS}=0.17 \pm 0.10 \mathrm{~mm}^{2}$ ) after 1 week. Interestingly, fibrin matrices decorated with both TG-VEGF and TG-PDGF-BB induced smaller scars than fibrin alone or with TG-VEGF alone.Quantification after 4 weeks confirmed similar results (Fig. 2D, Ctr $=0.97 \pm 0.33, V=1.08 \pm 0.25, V P=0.55 \pm 0.13$ vs PBS $\left.=0.19 \pm 0.14 \mathrm{~mm}^{2}\right)$. These results suggest that scar formation is not determined by injection trauma, but rather by the presence of fibrin itself.

The most prevalent cause of cardiac scarring is the sequelae of myocardial infarction. The early stages of scar formation after infarction comprise an inflammatory phase (up to 3 days), characterized by invasion of the damaged area by PDGF-R $\alpha+$ inflammatory fibroblasts, and a proliferative phase (until 7-14 days), during which activated fibroblasts upregulate $\alpha$-SMA expression, convert to myofibroblasts and start collagen deposition (Talman \& Ruskoaho, 2016). Upon injection, the hydrogel is acellular and therefore any cell detected inside it during the first few hoursrepresents the early response of the myocardium to the gel.Three hours after fibrin injection it can be seen that the first cells infiltrating the hydrogels are macrophages (Fig. 3A), marked by the specific expression of CD206, which is highly expressed both in cardiac resident and post-infarction activated macrophages(Shiraishi et al., 2016).Similarly to the evolution of post-infarction scar formation, fibrin hydrogels were progressively invaded by PDGF-R $\alpha+$ cardiac fibroblasts (Kanisicak et al., 2016) over the first 5 days, which subsequently converted to $\alpha$-SMA+ myofibroblasts(Fu et al., 2018) with a maximum frequency by 7 days after injection. 


\section{Scar formation is independent of adaptive immune response}

Next, we sought to determine whether scar formation might be promoted by an immune response against the hydrogel components of human origin, i.e. fibrinogen and the crosslinking enzymes.Therefore, both empty and VEGF-decorated fibrin hydrogels were injected in the left ventricle myocardium of nu/nu nude rats, which lack T lymphocytes and cannot mount an adaptive immune response, providing atolerogenic environment to xenogenic cells and proteins. As a further control, hearts were implanted also with human adiposederived mesenchymal cells (ASC) alone or over-expressing VEGF after genetic modification with a retroviral vector (L. F. Melly et al., 2012).Quantification of sections stained with Masson Trichrome confirmed that both fibrin groups induced significantly larger scars than the cell groups, regardless of VEGF presence(Fig. $4 A-B ; C$ Ctrgel $=1.85 \pm 0.50$ and $\mathrm{V}$ gel $=$ $1.61 \pm 0.40 \mathrm{~mm}^{2}$ vs Ctrcells $=0.74 \pm 0.25$ and $V$ cells $=1.08 \pm 0.31 \mathrm{~mm}^{2}$ ). Interestingly, immunofluorescence staining for endothelium (CD31) and smooth muscle cells ( $\alpha$-SMA) showed that cell-basedVEGF expression could induce effective angiogenesis at the border of the injection area, as previously reported (L. F. Melly et al., 2012), in contrast with the VEGFdecorated fibrin hydrogels (Fig. 4C). These results show that fibrin-associated scar formation is independent of adaptive immune response and that itprevents VEGF-induced myocardial angiogenesis.

\section{Scar formation is independent of hydrogel volume or mechanical stiffness}

Lastly, we investigated whether scar formation may correlate with mechanical trauma to the tissue due to the presence of the solid hydrogel in the actively contracting myocardium. Therefore we evaluated the effects on scar formation of reducing mechanical disturbance to the tissue by changing the size or the stiffness (fibrinogen concentration) of the hydrogels: 
a) a 2.5-fold smaller volume (10 $\mu \mathrm{l})$ without changing hydrogel composition(Vol); or b) a 10fold lower concentration offibrinogen $(2.5 \mathrm{mg} / \mathrm{ml})$ without changing injection volume (Fib). Quantification of tissue sections after 4 weeks showed that neither smaller nor softer hydrogels reduced the scar size compared to the control injectionsof $25 \mu$ lwith a fibrinogen concentration of $25 \mathrm{mg} / \mathrm{ml}\left(\mathrm{Ctr}=0.79 \pm 0.28, \mathrm{Vol}=0.91 \pm 0.19, \mathrm{Fib}=0.93 \pm 0.23 \mathrm{~mm}^{2}, \mathrm{p}=\right.$ n.s. $)$

(Fig. 5).

\section{Fibrin hydrogelpersistence increases scar formation}

Taken together, these data indicate that scar formation is induced exclusively by the presence of fibrin itself. Therefore, we sought to confirm this finding by the opposite approach and investigated whether prolonging fibrin persistence in the myocardium could increase scar size.First we labelled the injected hydrogels with fluorescent fibrinogen in order to track its degradation kinetics in the myocardium. As shown in Fig. 6A, fibrin is clearly visible and it is rapidly degraded during the first few days after injection, until traces can still be found at day5 and it is completely absent by day 7. Hydrogel duration was increased by incorporating $56 \mu \mathrm{g} / \mathrm{ml}$ of the fibrinolysis inhibitor aprotinin. In fact, although hydrogel degradationis influenced to some extent by fibrin concentration, pharmacologic inhibition of fibrinolysis by aprotinin incorporation has been shown previously to significantly delayfibrin degradation and prolong hydrogel persistence up to 4 weeks in skeletal muscle (Sacchi et al., 2014). As shown in Fig. 6B-C, after three weeks aprotinincontaining hydrogels caused significantlylarger scars than the fast-degrading controls(Ctr = $0.81 \pm 0.34$ vsApro $\left.=1.81 \pm 0.56 \mathrm{~mm}^{2}, \mathrm{p}<0.01\right)$. 


\section{Discussion}

Fibrin is widely used in clinical surgery and provides an attractive platform for the controlled delivery of therapeutic factors, thanks to the development of protein engineering strategies that enable their crosslinking to fibrinogen by coagulation factor XIII and therefore the decoration of the resulting matrix with desired doses and combinations of factors (Martino et al., 2015). Here we identified a serious side-effect of fibrin-based factor delivery to the myocardium, on one hand causing the formation of large fibrous scars, and on the other jeopardizing the therapeutic effect of delivered factors. Scar formation was not due to tissue damage associated with injection of the hydrogel, its mechanical stiffness or volume, nor to an immune reaction against the human components. Rather, the presence of fibrin per se was sufficient to kick-off the myocardial scarring response. Interestingly, the process appeared to be irreversible, since fibrin was degraded within 5 days in the myocardium, but the resulting scars persisted at least 4 weeks without showing signs of resolution.

A few groups described previously the intra-myocardial injection of different cellular therapies embedded in fibrin hydrogels and did not report a specific fibrotic response (Christman, Fok, et al., 2004; Christman, Vardanian, et al., 2004; Ryu et al., 2005).Epicardial patching with cell-seeded fibrin-based scaffolds has also been reported with similar results (Godier-Furnemont et al., 2011; Xiong et al., 2011; Ye et al., 2014).However, it should be noted that all these studies were performed in the presence of cardiac injury, ischemic or otherwise, with significant baseline scarring, making it difficult to identify a possible contribution by the fibrin component. The experiments reported here were instead performed in the intact myocardium of healthy animals in order to avoid confounding factors of underlying injury. 
Injection of a hydrogel in the myocardium is not a mechanically-neutralprocedure, as the material occupies some space and persists for a certain amount of time. However, several lines of evidence suggest that mechanical disturbance does not play a crucial rolein the observed fibrin-induced fibrotic scar formation: 1) the fibrinogen solution is liquid and takes over1 minute to polymerize. Therefore the hydrogel does not form a solid bolus, but rather spreads fully in the interstitial spacesas a sparse network of thin threads, which are only micron-thick and do not disturb the tissue integrity of the myocardium, as can be seen in Fig. $6 \mathrm{~A}$ and in greater magnification in Fig. $3 \mathrm{~A} ; 2$ ) Despite reducing the injected volume by $60 \%$, the amount of scarring induced was not diminished at all, not even as a non-significant trend (Fig. 5B); 3) Reducingthe fibrinogen concentration by 10 -fold generates significantly softer gels, similar to natural blood clots, but again no reduction in the scar size was observed; 4) Even the full-concentration fibrin hydrogels used here are significantly softer $(<3 \mathrm{KPa})$ than healthy myocardium matrix (>20 KPa)(Gaetani et al., 2020).

The observed scar formation is not a universal property of fibrin, but rather a tissuespecific effect. In fact, the same fibrin-based platform was previously investigated to deliver controlled doses of TG-VEGF in both normal and ischemic skeletal muscle or to ischemic skin wounds in mice, and in both tissues it induced robust and functional angiogenesis without any sign of scar or fibrosis, restored blood flow and promoted healing (Sacchi et al., 2014). Fibrin has been also extensively investigated as a delivery system for growth factors in wound healing, both exploiting its natural binding for a variety of therapeutic factors (Martino, Briquez, Ranga, Lutolf, \& Hubbell, 2013) and with different modifications to tailor factor release (Whelan, Caplice, \& Clover, 2014) and no skin fibrosis has been observed. Bladder smooth muscle has also been targeted with a bioactive fibrin-based bulking material to treat urinary incontinence, without any report of ensuing fibrosis(Vardar et al., 
2019). Further, fibrin has no direct toxic effect on cardiomyocytes, as it is widely used to generate engineered heart tissues and cardiac patches with both native and iPSC-derived cardiomyocytes (reviewed in (Roura, Galvez-Monton, \& Bayes-Genis, 2017)).

The results reported here were obtained in the normal myocardium of healthy animals and therefore are not directly translatable to the pathogenesis of specific cardiac injury processes. However, since fibrotic scarring is a common end-point of a variety of myocardial injuries, it is useful to draw some comparison with what is known about this process, for example in the best studied pathological condition, i.e. myocardial infarction. Upon ischemia or other kinds of injury, cardiomyocyte death rapidly activates innate immune pathways that trigger cytokine, chemokine and adhesion molecule expression, initiating the inflammatory phase that subsequently evolves into fibrosis(Kong, Christia, \& Frangogiannis, 2014). Taken together our results show that fibrin per se is capable of startinga similar scarringprocess. In fact, fibrous scars were induced also when fibrinogen concentration was reduced by 10 -fold, which is in the range of a physiological blood clot (Bale, Muller, \& Ferry, 1985). The addition of VEGF alone did not reduce the scar. However, when PDGF-BB was added to VEGF, a positive trend toward a smaller scar was observed. This finding is compatible with the activation of thePGDF-Receptor $\beta$ (PDGF-Rß) signaling pathway in the perivascular and mononuclear cells that takes place also after myocardial infarction(Zymek et al., 2006). Whereas PDGF-R $\alpha$ signaling does not regulate vascular maturation, it promotes collagen deposition (Zymek et al., 2006). On the contrary, the activation of PDGF-R $\beta$ plays a crucial role in vascular maturation by regulating the recruitment of mural cells to growing microvessels, and its inhibition by aneutralizing antibody critically impaired vascular maturation in healing infarcts (Sano et al., 2001). The role of PDGF-R $\beta$ signaling in healing infarcts likely involves a paracrine interaction between endothelial cells that secrete PDGF- 
BB and mural cells like pericytes andsmooth muscle cellsthat express the receptor (Hellstrom, Kalen, Lindahl, Abramsson, \& Betsholtz, 1999).

Fibrous tissue accumulation relies on the balance between collagen synthesis and degradation. Collagen synthesis is primarily stimulated by Transforming Growth Factor- $\beta$ (TGF- $\beta$ ), whereas matrix metallo-proteases are central to collagen degradation (Talman \& Ruskoaho, 2016). Myofibroblasts play a key role in fibrous tissue formation, for example in the infarcted myocardium, as they are not present in the normal myocardium, but accumulate after injury(Fu et al., 2018). During cardiac remodeling, fibroblasts differentiate into myofibroblasts, which are fast-proliferating, $\alpha$-smooth muscle cell actin ( $\alpha$-SMA)positive cells with pronounced contractile and secretory properties (Tomasek, Gabbiani, Hinz, Chaponnier, \& Brown, 2002). After depositing collagen in the normal repair process myofibroblasts undergo apoptosis, but if this does not occur then their persistent activity causes excessive matrix accumulation and detrimental fibrosis (Shamhart \& Meszaros, 2010). Myofibroblasts can also be induced by TGF- $\beta$ itself and lead to excessive matrix accumulation (van den Borne et al., 2010). TGF- $\beta$ plays therefore an important role in modulating fibroblast phenotype towards fibrosis gene expression, and in promoting extracellular matrix deposition in the infarct region (Bujak \& Frangogiannis, 2007).

Interestingly, fibrinogen has been recently found to trigger astrocyte scar formation in the central nervous system through TGF- $\beta$ signaling (Schachtrup et al., 2010). Fibrinogen is one of the first blood proteins that leaks out of broken vessels immediately after injury and it acts as a multi-faceted signaling molecule by interacting with integrins and non-integrin receptors, as well as by functioning as a carrier of growth factors and regulator of their bioavailability(Petersen, Ryu, \& Akassoglou, 2018). In a murine model of traumatic injury to 
the brain cortex, genetic or pharmacologic depletion of fibrinogen prevented astrocytic scar formation, whereas the simple injection of fibrinogen into the cortex induced scarring even in the absence of trauma (Schachtrup et al., 2010). Mechanistically, fibrinogen-bound latent TGF- $\beta$ was found to interact with local perivascular astrocytes, leading to active TGF- $\beta$ formation, which induces reactive astrocytosis by regulating the TGF- $\beta / \mathrm{Smad}$ signaling pathway, resulting in scar formation(Schachtrup et al., 2010). Local provisional fibrin matrix thus functions as a primary astrocyte activation signal initiating scar formation in the CNS by regulating the bioavailability of active TGF- $\beta$ at sites of vascular damage.Based on the function of fibrinogen to activate TGF- $\beta$ signaling and to promote astrocytic gliosis in the central nervous system, it is tempting to speculate that a similar mechanism might underlie the ability of fibrinogen to induce fibrotic scarring in the myocardium that we identified here.

Several lines of evidence suggest that a complex interaction involving TGF- $\beta$, Endothelin1 (ET-1) and the renin-angiotensin-aldosterone system cause fibrogenesis (Leask, 2015). Angiotensin-II (Ang-II) causes vaso-constriction and its circulating concentration is elevated in patients with fibrotic hearts (Schnee \& Hsueh, 2000). In rats with myocardial fibrosis, AngII is both expressed and activated by macrophages and myofibroblasts (Sun, Cleutjens, DiazArias, \& Weber, 1994).In cardiac fibroblasts, Ang-II induces expression of collagen through TGF- $\beta /$ Smad3 and extracellular signal-regulated kinase by an interleukin 6 (IL-6)-dependent mechanism. Angiotensin receptor inhibitors, such as losartan, are effective in reducing cardiac fibrosis in both animals and humans (Leask, 2015).Losartan is believed to reduce cardiac fibrosis through the inhibition of the endothelial to mesenchymal transition, as was demonstrated in mitral valve endothelial cells, by acting to block the Angll-elicited, TGF- $\beta$ induced phosphorylation of ERK1/2 (Oliveira-Junior et al., 2014). Losartan also reduces 
collagen I synthesis and fibroblast activation in response to stimulation by Angll(Galie, Russell, Westfall, \& Stegemann, 2012). Several other pathways are playing a role in activating TGF- $\beta$ and are currently under investigation in clinical trials. Spironolactone, a specific pharmacologic antagonist of aldosterone, seems to have an influence on serum markers of collagen metabolism and on other cardiovascular structures (Pellicori et al., 2020). Perfinidone, a broad anti-inflammatory drug, has an effect of blocking TGF- $\beta$ and Ang-II-induced fibrosis by controlling the feedback loop between the Ang-II type 1 receptor, the phsoph-p38 mitogen-activated protein kinase pathway and the renin-angiotensin system, which provides its cardio-protective effect (Li et al., 2017). TGF- $\beta$ and hypoxia are important stimuli for the expression of the collagen cross-linking enzymelysil oxidase (LOX) in a number of cells and tissues, and its inhibition with beta-amino-proprionitrile (BAPN) resulted in a significant reduction in both collagen cross-linking and content (GonzalezSantamaria et al., 2016). Further, it should also be noted that the transglutaminase activity of Factor XIIla, which is required for the formation of fibrin hydrogel, is not specific for fibrinogen and has the ability to cross-link a wide range of substrates, including several extracellular matrix proteins like fibronectin and vitronectin (Dickneite et al., 2015). It is unclear whether this enzymatic activity in the myocardium ECM may also have a role in causing the observed scar formation.

In conclusion intramyocardial delivery of growth factors by fibrin-based matrices poses significant risks of unwanted fibrosis. Future studies should aim at dissecting the mechanisms underlying the promotion of myocardial fibrotic scar formation by fibrin hydrogels, including the possible roles of fibrinogen monomers, the transglutaminase FXIIlaandthe TGF- $\beta$ pathway, to overcome the scarring process and enable the exploitation of this attractive therapeutic platform for recombinant growth factor delivery. 


\section{Authorship}

LM, AG,CS,NDM, BRandAB conceivedand designedthe experiments.LM, AG, AMandNDMacquired data.LM, AG, CSP, CYH, MCNandCSanalyzedand interpreted the data.LM, CS, BRandAB drafted the work.AG, CSP, CYH, MCN, AM and NDMrevised critically the manuscriptfor important intellectual content.All co-authors gave final approvalofthesubmitted manuscript.

\section{Acknowledgments}

We gratefully acknowledge Ms. M. Ast-Dumbach and Mr. P. Thurionfor their expertise in histology and their technical assistance with tissue staining. This work was supported in part by an intramural grant of the CHU UCL Namur (Mont Godinne Foundation) to L.M., as well asgrants by the Swiss National Science Foundation (163202)and the European Union FP7 (ANGIOSCAFF, CP-IP 214402) to A.B.

\section{Conflict of interest}

None to declare. 


\section{References}

Annex, B. H. (2013). Therapeutic angiogenesis for critical limb ischaemia. Nat Rev Cardiol, 10(7), 387-396. doi:10.1038/nrcardio.2013.70

Bale, M. D., Muller, M. F., \& Ferry, J. D. (1985). Rheological studies of creep and creep recovery of unligated fibrin clots: comparison of clots prepared with thrombin and ancrod. Biopolymers, 24(3), 461-482. doi:10.1002/bip.360240304

Baum, C., Dullmann, J., Li, Z., Fehse, B., Meyer, J., Williams, D. A., \& von Kalle, C. (2003). Side effects of retroviral gene transfer into hematopoietic stem cells. Blood, 101(6), 2099-2114. doi:10.1182/blood-2002-07-2314

Bujak, M., \& Frangogiannis, N. G. (2007). The role of TGF-beta signaling in myocardial infarction and cardiac remodeling. Cardiovasc Res, 74(2), 184-195. doi:10.1016/j.cardiores.2006.10.002

Carmeliet, P., \& Jain, R. K. (2011). Molecular mechanisms and clinical applications of angiogenesis. Nature, 473(7347), 298-307. doi:10.1038/nature10144

Christman, K. L., Fok, H. H., Sievers, R. E., Fang, Q., \& Lee, R. J. (2004). Fibrin glue alone and skeletal myoblasts in a fibrin scaffold preserve cardiac function after myocardial infarction. Tissue Eng, 10(3-4), 403-409. doi:10.1089/107632704323061762

Christman, K. L., Vardanian, A. J., Fang, Q., Sievers, R. E., Fok, H. H., \& Lee, R. J. (2004). Injectable fibrin scaffold improves cell transplant survival, reduces infarct expansion, and induces neovasculature formation in ischemic myocardium. $\mathrm{J} \mathrm{Am}$ Coll Cardiol, 44(3), 654-660. doi:10.1016/j.jacc.2004.04.040

Dickneite, G., Herwald, H., Korte, W., Allanore, Y., Denton, C. P., \& Matucci Cerinic, M. (2015). Coagulation factor XIII: a multifunctional transglutaminase with clinical potential in a range of conditions. Thromb Haemost, 113(4), 686-697. doi:10.1160/TH14-07-0625

Fu, X., Khalil, H., Kanisicak, O., Boyer, J. G., Vagnozzi, R. J., Maliken, B. D., . . Molkentin, J. D. (2018). Specialized fibroblast differentiated states underlie scar formation in the infarcted mouse heart. J Clin Invest, 128(5), 2127-2143. doi:10.1172/JCI98215

Gaetani, R., Zizzi, E. A., Deriu, M. A., Morbiducci, U., Pesce, M., \& Messina, E. (2020). When Stiffness Matters: Mechanosensing in Heart Development and Disease. Front Cell Dev Biol, 8, 334. doi:10.3389/fcell.2020.00334

Galie, P. A., Russell, M. W., Westfall, M. V., \& Stegemann, J. P. (2012). Interstitial fluid flow and cyclic strain differentially regulate cardiac fibroblast activation via AT1R and TGF-beta1. Exp Cell Res, 318(1), 75-84. doi:10.1016/j.yexcr.2011.10.008 
Gianni-Barrera, R., Burger, M., Wolff, T., Heberer, M., Schaefer, D. J., Gurke, L., . . Banfi, A. (2016). Long-term safety and stability of angiogenesis induced by balanced single-vector co-expression of PDGF-BB and VEGF164 in skeletal muscle. Sci Rep, 6, 21546. doi:10.1038/srep21546

Gianni-Barrera, R., Di Maggio, N., Melly, L., Burger, M. G., Mujagic, E., Gurke, L., .. Banfi, A. (2020). Therapeutic vascularization in regenerative medicine: Concise Review. Stem Cells Transl Med. doi:10.1002/sctm.19-0319

Godier-Furnemont, A. F., Martens, T. P., Koeckert, M. S., Wan, L., Parks, J., Arai, K., . . . Vunjak-Novakovic, G. (2011). Composite scaffold provides a cell delivery platform for cardiovascular repair. Proc Natl Acad Sci U S A, 108(19), 7974-7979. doi:10.1073/pnas.1104619108

Gonzalez-Santamaria, J., Villalba, M., Busnadiego, O., Lopez-Olaneta, M. M., Sandoval, P., Snabel, J., . . . Rodriguez-Pascual, F. (2016). Matrix cross-linking lysyl oxidases are induced in response to myocardial infarction and promote cardiac dysfunction. Cardiovasc Res, 109(1), 67-78. doi:10.1093/cvr/cvv214

Hellstrom, M., Kalen, M., Lindahl, P., Abramsson, A., \& Betsholtz, C. (1999). Role of PDGF$B$ and PDGFR-beta in recruitment of vascular smooth muscle cells and pericytes during embryonic blood vessel formation in the mouse. Development, 126(14), 3047-3055. Retrieved from https://www.ncbi.nlm.nih.gov/pubmed/10375497

Kanisicak, O., Khalil, H., Ivey, M. J., Karch, J., Maliken, B. D., Correll, R. N., . . Molkentin, J. D. (2016). Genetic lineage tracing defines myofibroblast origin and function in the injured heart. Nat Commun, 7, 12260. doi:10.1038/ncomms12260

Kong, P., Christia, P., \& Frangogiannis, N. G. (2014). The pathogenesis of cardiac fibrosis. Cell Mol Life Sci, 71(4), 549-574. doi:10.1007/s00018-013-1349-6

Leask, A. (2015). Getting to the heart of the matter: new insights into cardiac fibrosis. Circ Res, 116(7), 1269-1276. doi:10.1161/CIRCRESAHA.116.305381

Li, C., Han, R., Kang, L., Wang, J., Gao, Y., Li, Y., ... Tian, J. (2017). Pirfenidone controls the feedback loop of the AT1R/p38 MAPK/renin-angiotensin system axis by regulating liver $\mathrm{X}$ receptor-alpha in myocardial infarction-induced cardiac fibrosis. Sci Rep, 7, 40523. doi:10.1038/srep40523

Martino, M. M., Briquez, P. S., Ranga, A., Lutolf, M. P., \& Hubbell, J. A. (2013). Heparinbinding domain of fibrin(ogen) binds growth factors and promotes tissue repair when incorporated within a synthetic matrix. Proc Natl Acad Sci U S A, 110(12), 4563-4568. doi:10.1073/pnas.1221602110

Martino, M. M., Brkic, S., Bovo, E., Burger, M., Schaefer, D. J., Wolff, T., ... Banfi, A. (2015). Extracellular matrix and growth factor engineering for controlled angiogenesis in regenerative medicine. Front Bioeng Biotechnol, 3, 45. doi:10.3389/fbioe.2015.00045 
Melly, L., Cerino, G., Frobert, A., Cook, S., Giraud, M. N., Carrel, T., . . Banfi, A. (2018). Myocardial infarction stabilization by cell-based expression of controlled Vascular Endothelial Growth Factor levels. J Cell Mol Med, 22(5), 2580-2591. doi: $10.1111 /$ jcmm.13511

Melly, L. F., Marsano, A., Frobert, A., Boccardo, S., Helmrich, U., Heberer, M., . . Banfi, A. (2012). Controlled angiogenesis in the heart by cell-based expression of specific vascular endothelial growth factor levels. Hum Gene Ther Methods, 23(5), 346356. doi:10.1089/hgtb.2012.032

Misteli, H., Wolff, T., Fuglistaler, P., Gianni-Barrera, R., Gurke, L., Heberer, M., \& Banfi, A. (2010). High-throughput flow cytometry purification of transduced progenitors expressing defined levels of vascular endothelial growth factor induces controlled angiogenesis in vivo. Stem Cells, 28(3), 611-619. doi:10.1002/stem.291

Oliveira-Junior, S. A., Martinez, P. F., Guizoni, D. M., Campos, D. H., Fernandes, T., Oliveira, E. M., . . . Cicogna, A. C. (2014). AT1 receptor blockade attenuates insulin resistance and myocardial remodeling in rats with diet-induced obesity. PLoS One, 9(1), e86447. doi:10.1371/journal.pone.0086447

Ozawa, C. R., Banfi, A., Glazer, N. L., Thurston, G., Springer, M. L., Kraft, P. E., . . Blau, H. M. (2004). Microenvironmental VEGF concentration, not total dose, determines a threshold between normal and aberrant angiogenesis. J Clin Invest, 113(4), 516527. doi:10.1172/JCI18420

Pellicori, P., Ferreira, J. P., Mariottoni, B., Brunner-La Rocca, H. P., Ahmed, F. Z., Verdonschot, J., . . . Cleland, J. G. F. (2020). Effects of spironolactone on serum markers of fibrosis in people at high risk of developing heart failure: rationale, design and baseline characteristics of a proof-of-concept, randomised, precisionmedicine, prevention trial. The Heart OMics in AGing (HOMAGE) trial. Eur J Heart Fail. doi:10.1002/ejhf.1716

Petersen, M. A., Ryu, J. K., \& Akassoglou, K. (2018). Fibrinogen in neurological diseases: mechanisms, imaging and therapeutics. Nat Rev Neurosci, 19(5), 283-301. doi:10.1038/nrn.2018.13

Roura, S., Galvez-Monton, C., \& Bayes-Genis, A. (2017). Fibrin, the preferred scaffold for cell transplantation after myocardial infarction? An old molecule with a new life. J Tissue Eng Regen Med, 11(8), 2304-2313. doi:10.1002/term.2129

Ryu, J. H., Kim, I. K., Cho, S. W., Cho, M. C., Hwang, K. K., Piao, H., . . Kim, B. S. (2005). Implantation of bone marrow mononuclear cells using injectable fibrin matrix enhances neovascularization in infarcted myocardium. Biomaterials, 26(3), 319326. doi:10.1016/j.biomaterials.2004.02.058

Sacchi, V., Mittermayr, R., Hartinger, J., Martino, M. M., Lorentz, K. M., Wolbank, S., . . . Banfi, A. (2014). Long-lasting fibrin matrices ensure stable and functional 
angiogenesis by highly tunable, sustained delivery of recombinant VEGF164. Proc Natl Acad Sci U S A, 111(19), 6952-6957. doi:10.1073/pnas.1404605111

Sano, H., Yokode, M., Takakura, N., Takemura, G., Doi, T., Kataoka, H., . . Kita, T. (2001). Study on PDGF receptor beta pathway in glomerular formation in neonate mice. Ann N Y Acad Sci, 947, 303-305. doi:10.1111/j.1749-6632.2001.tb03951.x

Schachtrup, C., Ryu, J. K., Helmrick, M. J., Vagena, E., Galanakis, D. K., Degen, J. L., . . . Akassoglou, K. (2010). Fibrinogen triggers astrocyte scar formation by promoting the availability of active TGF-beta after vascular damage. J Neurosci, 30(17), 5843-5854. doi:10.1523/JNEUROSCI.0137-10.2010

Schnee, J. M., \& Hsueh, W. A. (2000). Angiotensin II, adhesion, and cardiac fibrosis. Cardiovasc Res, 46(2), 264-268. doi:10.1016/s0008-6363(00)00044-4

Shamhart, P. E., \& Meszaros, J. G. (2010). Non-fibrillar collagens: key mediators of postinfarction cardiac remodeling? J Mol Cell Cardiol, 48(3), 530-537. doi:10.1016/j.yjmcc.2009.06.017

Shiraishi, M., Shintani, Y., Shintani, Y., Ishida, H., Saba, R., Yamaguchi, A., . . Suzuki, K. (2016). Alternatively activated macrophages determine repair of the infarcted adult murine heart. J Clin Invest, 126(6), 2151-2166. doi:10.1172/JCI85782

Sun, Y., Cleutjens, J. P., Diaz-Arias, A. A., \& Weber, K. T. (1994). Cardiac angiotensin converting enzyme and myocardial fibrosis in the rat. Cardiovasc Res, 28(9), 1423-1432. doi:10.1093/cvr/28.9.1423

Talman, V., \& Ruskoaho, H. (2016). Cardiac fibrosis in myocardial infarction-from repair and remodeling to regeneration. Cell Tissue Res, 365(3), 563-581. doi:10.1007/s00441-016-2431-9

Tomasek, J. J., Gabbiani, G., Hinz, B., Chaponnier, C., \& Brown, R. A. (2002). Myofibroblasts and mechano-regulation of connective tissue remodelling. Nat Rev Mol Cell Biol, 3(5), 349-363. doi:10.1038/nrm809

van den Borne, S. W., Diez, J., Blankesteijn, W. M., Verjans, J., Hofstra, L., \& Narula, J. (2010). Myocardial remodeling after infarction: the role of myofibroblasts. Nat Rev Cardiol, 7(1), 30-37. doi:10.1038/nrcardio.2009.199

Vardar, E., Vythilingam, G., Pinnagoda, K., Engelhardt, E. M., Zambelli, P. Y., Hubbell, J. A., . . Larsson, H. M. (2019). A bioactive injectable bulking material; a potential therapeutic approach for stress urinary incontinence. Biomaterials, 206, 41-48. doi:10.1016/j.biomaterials.2019.03.030

Whelan, D., Caplice, N. M., \& Clover, A. J. (2014). Fibrin as a delivery system in wound healing tissue engineering applications. J Control Release, 196, 1-8. doi:10.1016/j.jconrel.2014.09.023

Xiong, Q., Hill, K. L., Li, Q., Suntharalingam, P., Mansoor, A., Wang, X., . . Zhang, J. (2011). A fibrin patch-based enhanced delivery of human embryonic stem cell-derived 
vascular cell transplantation in a porcine model of postinfarction left ventricular remodeling. Stem Cells, 29(2), 367-375. doi:10.1002/stem.580

Ye, L., Chang, Y. H., Xiong, Q., Zhang, P., Zhang, L., Somasundaram, P., ... Zhang, J. (2014). Cardiac repair in a porcine model of acute myocardial infarction with human induced pluripotent stem cell-derived cardiovascular cells. Cell Stem Cell, 15(6), 750-761. doi:10.1016/j.stem.2014.11.009

Zymek, P., Bujak, M., Chatila, K., Cieslak, A., Thakker, G., Entman, M. L., \& Frangogiannis, N. G. (2006). The role of platelet-derived growth factor signaling in healing myocardial infarcts. J Am Coll Cardiol, 48(11), 2315-2323. doi:10.1016/j.jacc.2006.07.060

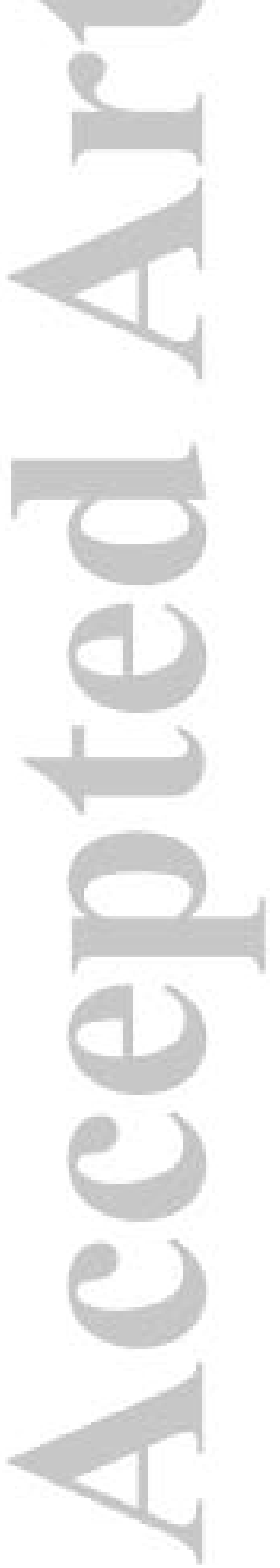


A
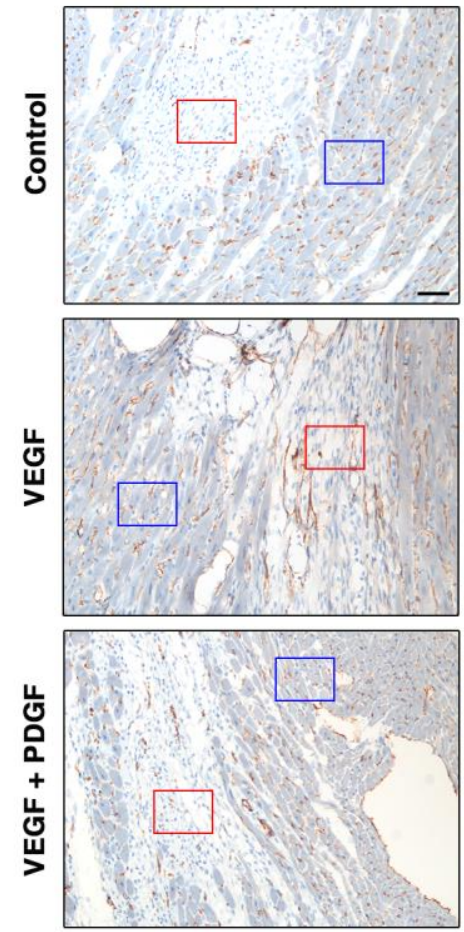

B

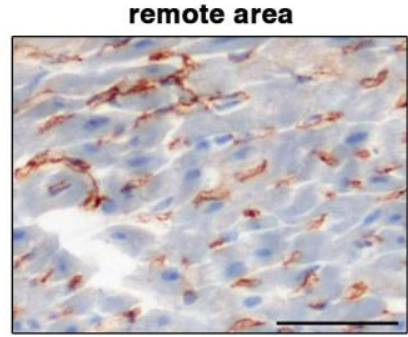

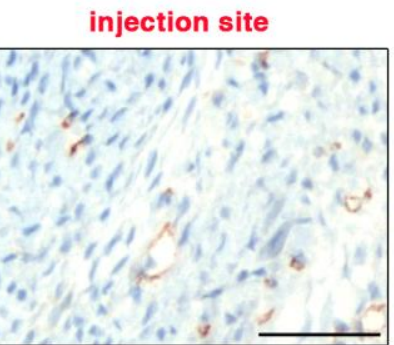
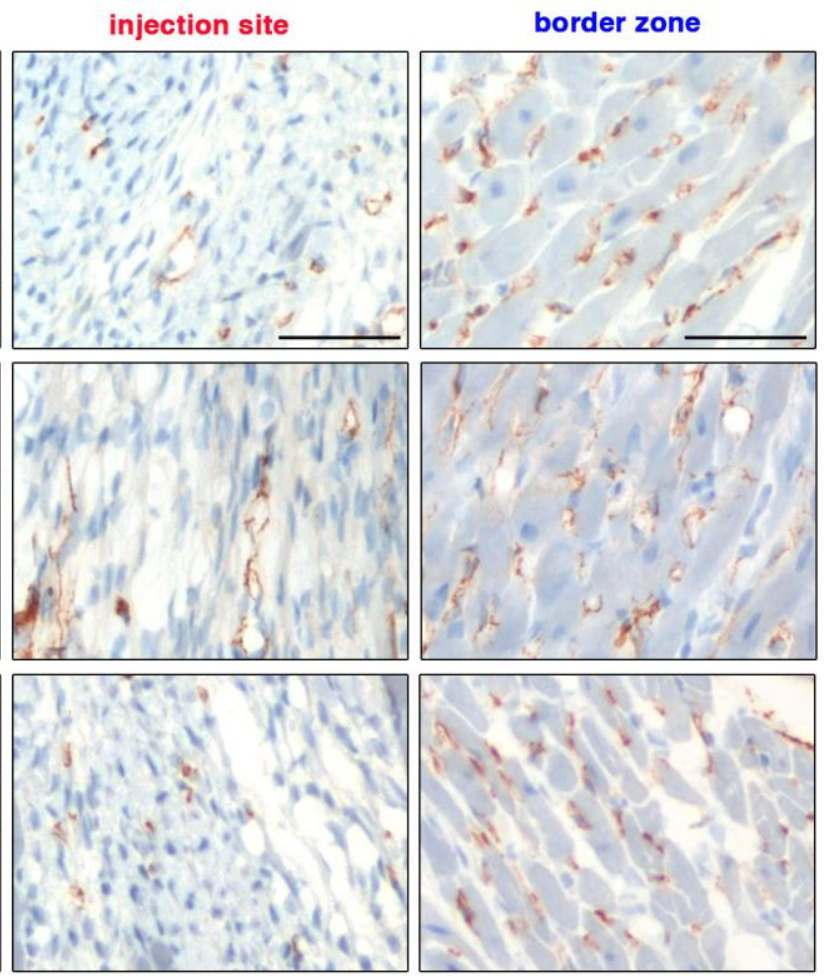

C

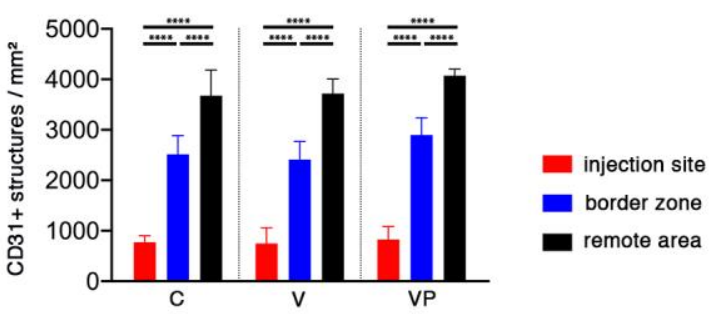

\section{Figure 1}

Figure 1. Absence of neo-angiogenesis.

(A) Angiogenesis analysis in immunohistochemistry (CD31, in brown) at one week after intramyocardial delivery of the hydrogel for all three treatment groups: empty gel, loaded with VEGF only and in combination with PDGF. (B) As negative controls, the remote areas in the posterior wall of the myocardium were used. Size bar $=50 \mu \mathrm{m}$. (C) Quantifications were made for all treatment groups ( $n=6$ for each group) within the injection site (red squares in $\mathrm{A})$, at the border zone (blue squares in A) and in a remote area (black). ${ }^{* * * *} \mathrm{P}<0.0001$. 

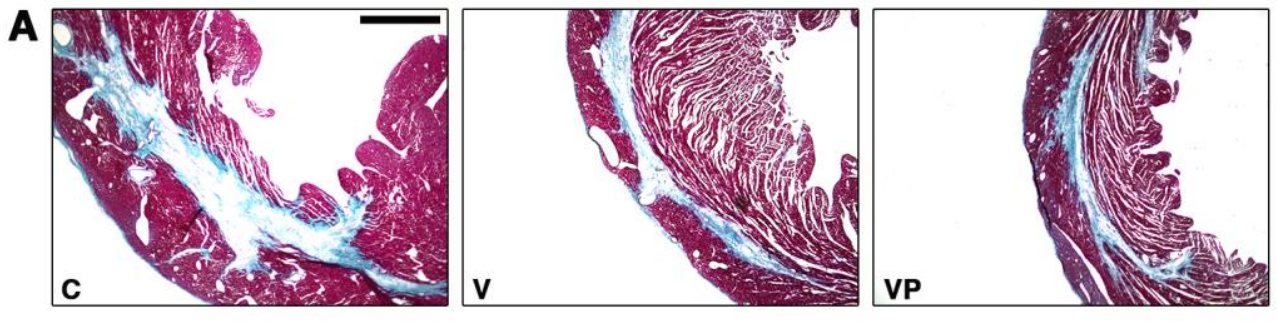

B

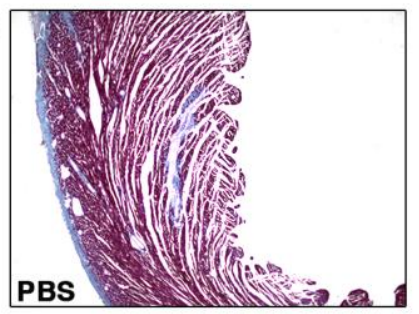

C

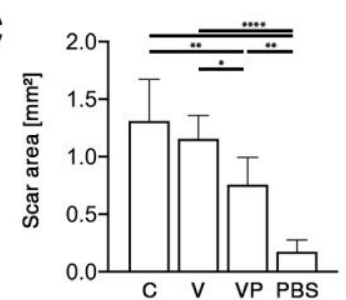

D

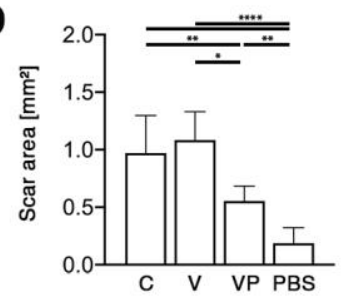

Figure 2

Figure 2. Presence of a fibrotic scar.

(A) Fibrosis analysis on Masson Trichrome sections for all three treatment groups. (B) A negative control with phosphate-buffered saline (PBS) injection was added. ). Size bar $=1$ $\mathrm{mm}$. Scar area was quantified at one (C) and four (D) weeks $(n=6)$. ${ }^{*} P<0.05,{ }^{* *} P<0.01$, $* * * * \mathrm{P}<0.0001$. 
A
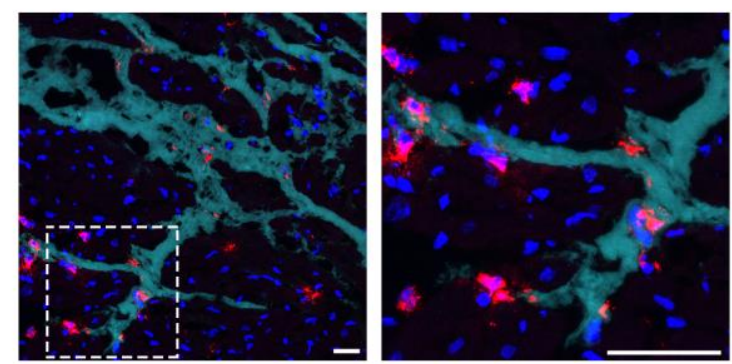

B

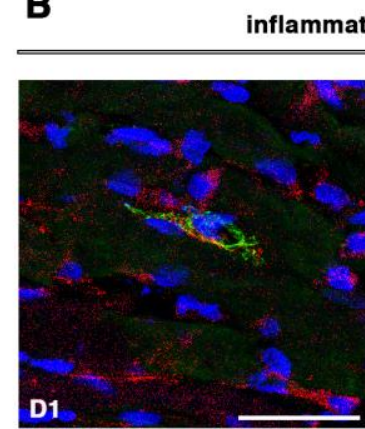

inflammatory phase
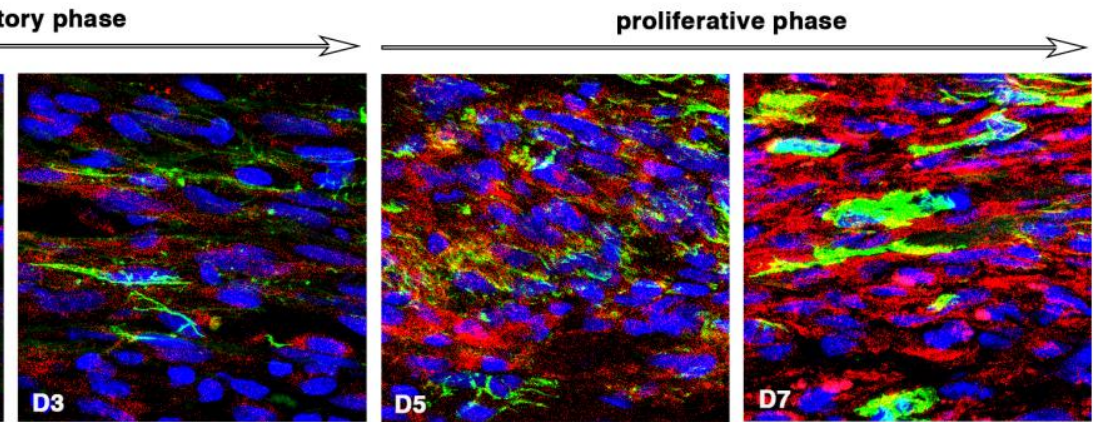

Figure 3

Figure 3. Cell population in the gel and in the injection site.

(A) Immunofluorescence 3 hours after injection with 647-labeled fibrinogen (in cyan) andstained for nuclei (DAPI, in blue) and the specific macrophage marker CD206(in magenta). The right panel provides a higher magnification view of the white square in the left panel.(B) Immunofluorescence for activated ( $\alpha$ SMA in green) cardiac fibroblasts (PDGFR $\alpha$ in red), and during the first week at days 1,3,5,7 (D1, D3, D5, D7). Size bars = $30 \mu \mathrm{m}$. 
A

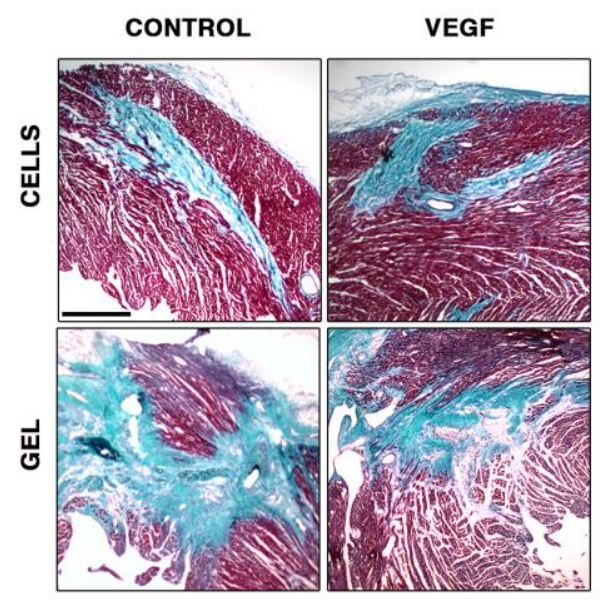

C

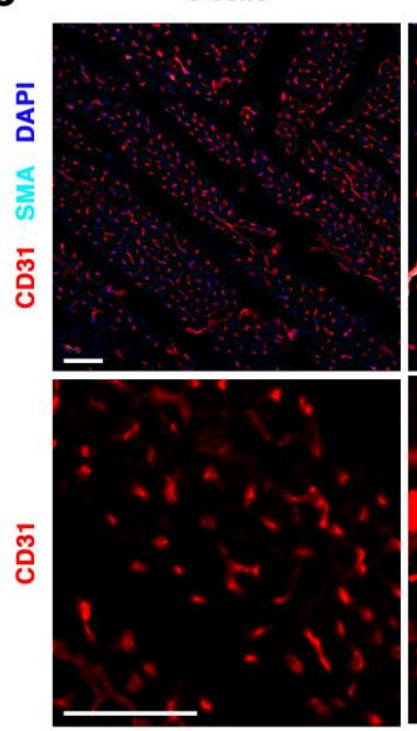

V cells

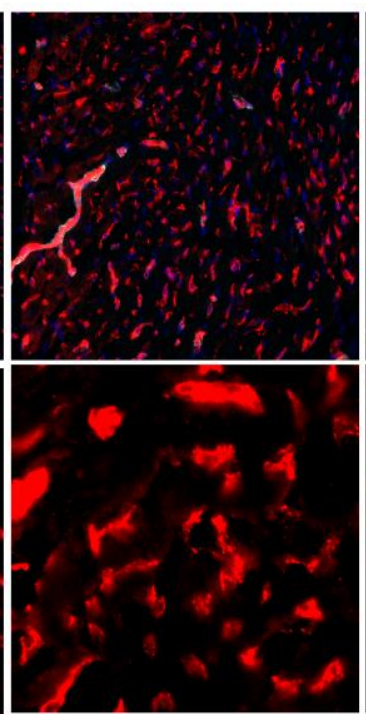

B

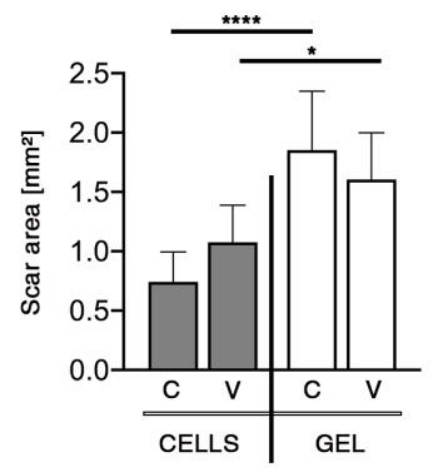

V gel
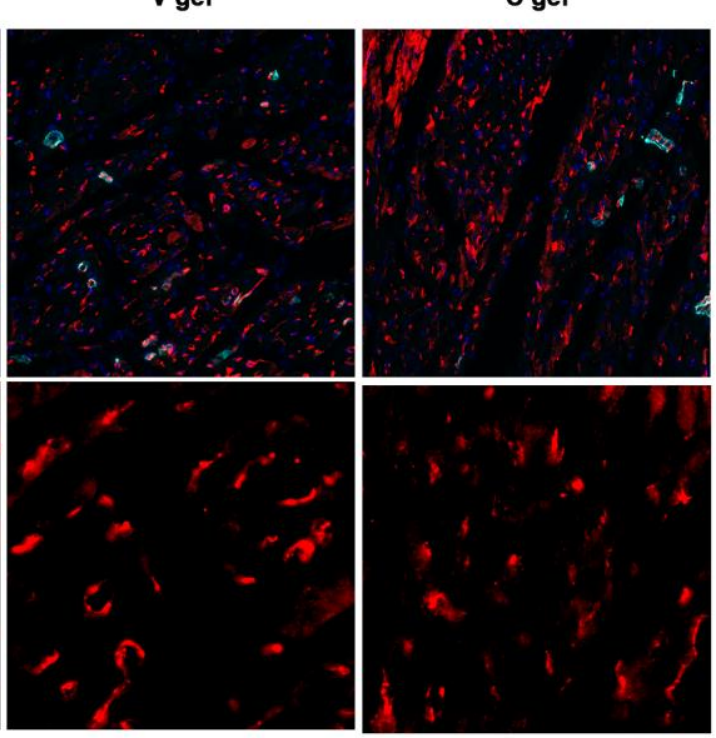

Figure 4

Figure 4. Effect of the immune response against fibrin and of the VEGF itself.

Effect of the gel on T-cell deficient animals with cell-based versus hydrogel-based delivery at four weeks. Adipose-derived mesenchymal stem cells alone (control cells) or transduced to express VEGF (VEGF cells), empty gel and/or loaded with VEGF. (A) Morphology of the scar in Masson Trichrome. (B) Scar area quantification at four weeks ( $n=6)$. Size bar $=1 \mathrm{~mm} .{ }^{*} P<$ $0.05, * * * * P<0.0001$. (C) Morphology of the angiogenesis in immunofluorescence staining for endothelium (CD 31, in red), for smooth muscle cells (SMA, in cyan) and nuclei (DAPI, in blue) and at higher magnification for CD31 only. Size bar $=50 \mu \mathrm{m}$. 


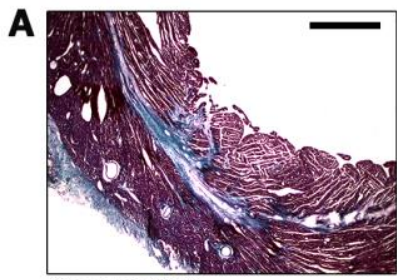

Control (Ctr)

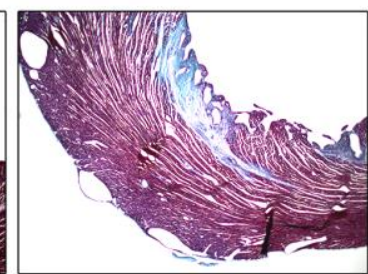

Low volume (Vol)

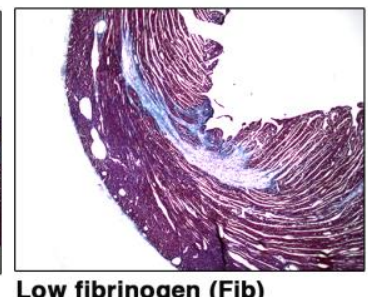

Low fibrinogen (Fib)

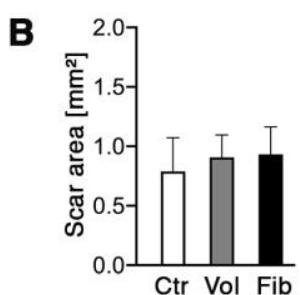

Figure 5

Figure 5. Effects of the volume injected and gel stiffness.

(A) Masson Trichrome after the injection of a reduced volume (Vol) of $10 \mu \mathrm{L}$ and of a 10 -fold reduced fibrinogen concentration of $2.5 \mathrm{mg} / \mathrm{mL}$ (Fib) compared to the control, (Ctr, injection of $25 \mathrm{uL}$ [Fibirnogen] of $25 \mathrm{mg} / \mathrm{mL}$ ). Size bar $=1 \mathrm{~mm}$.(B) Quantification of the scar area at 1

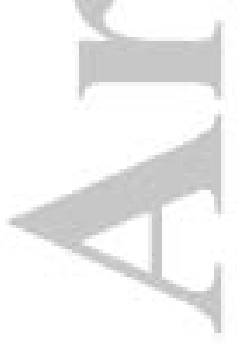
week $(n=6)$. 


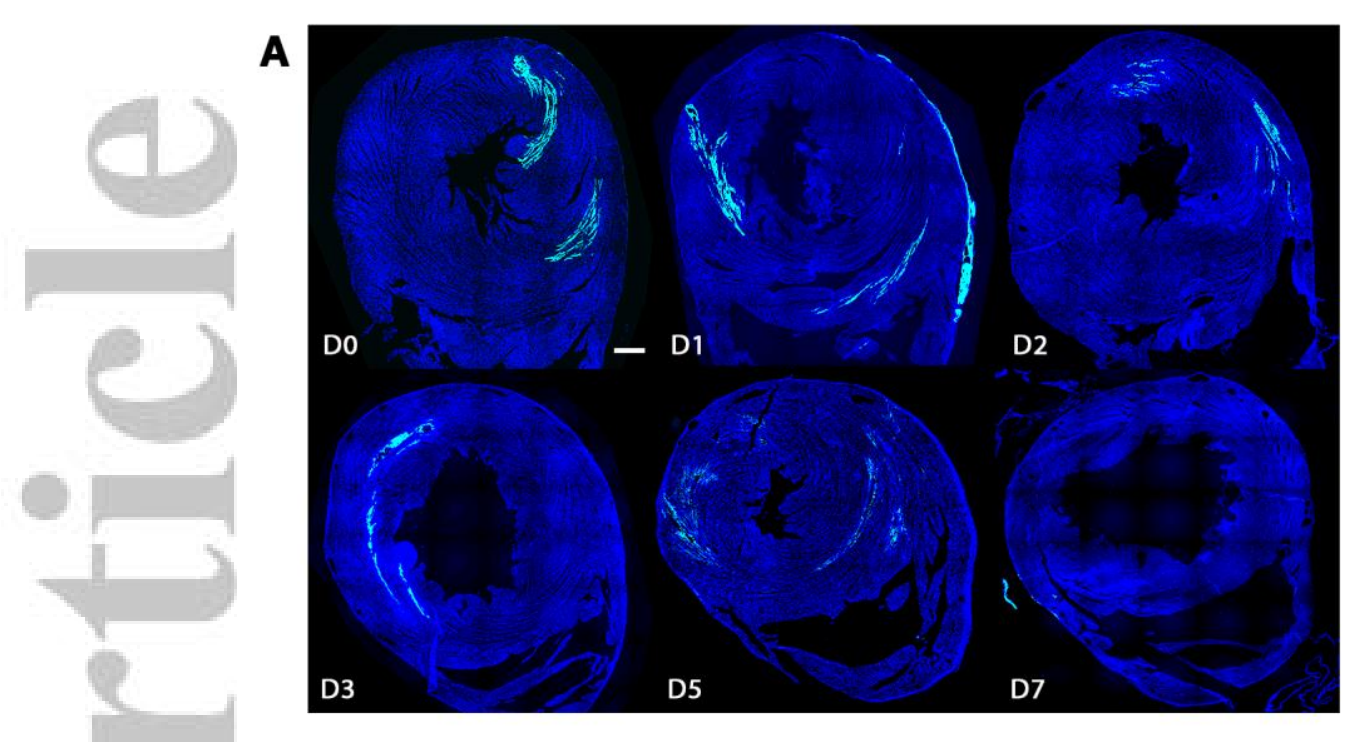

B

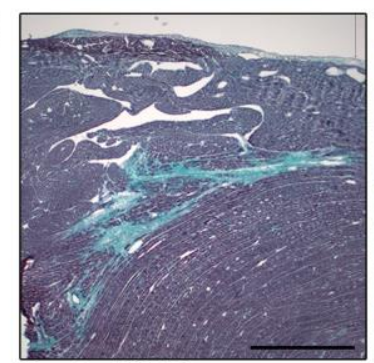

Control (Ctr)

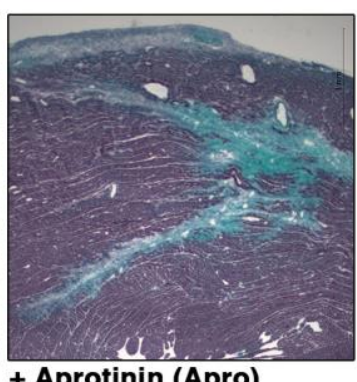

+ Aprotinin (Apro)
C

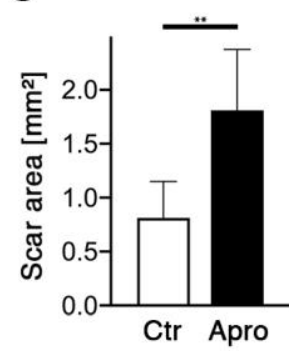

Figure 6

Figure 6. Influence of the gel degradation.

(A) Gel degradation for the first week in immunofluorescence with 647-labeld fibrinogen (in cyan) and nuclei (DAPI, in blue). Size bar $=1 \mathrm{~mm}$. (B) Aprotinin $(56 \mu \mathrm{g} / \mathrm{mL})$ was added to the hydrogel and the maximal scar area (C) was assessed at 3 weeks $(n=6) .{ }^{* *} P<0.01$. 